because there is no evidence that the intervention reduced suicidal behaviour, which was not measured as an outcome - not even through the obvious mechanism of recording re-attendance following non-fatal self-harm. Neither is it entirely accurate to imply, as the title does, that the intervention was designed to reduce suicidal behaviour. In the body of the paper, the authors indicate that they did not nominate a primary outcome measure because - in what they call an exploratory trial - they weren't clear what effects they expected.

Second, there is a stage at which flaws in the design or delivery of a trial mean that no safe conclusions can be drawn from it. Loss to follow-up of $50-60 \%$ at 3 months and $>85 \%$ at 6 months is not compatible with a plausible intention-to-treat analysis, and using last observation carried forward cannot rescue the situation - especially when (as is the case here) the last observations in question are pre-intervention baseline measures taken at the same time that participants were given the materials for the intervention to which they had been randomised. The authors indicate that they are aware of some of the other problems with their trial for example, outcomes not collected by researchers masked to allocation and uncorrected analysis of multiple outcomes. Under the circumstances, their conclusion that their intervention showed promise - a conclusion about which they were surely not in initial equipoise - cannot be regarded as sound.

1 Armitage CJ, Rahim WA, Rowe R, O'Connor RC. An exploratory randomised trial of a simple, brief psychological intervention to reduce subsequent suicidal ideation and behaviour in patients admitted to hospital for self-harm. Br J Psychiatry 2016; 208: 470-6.

Allan House, Professor of Liaison Psychiatry, University of Leeds. Email: a.o.house@leeds.ac.uk; David Owens, University of Leeds

doi: $10.1192 /$ bjp.209.4.350a

Authors' reply: We welcome the opportunity to reply to House \& Owens' two observations. We will address their concerns in the order in which they present them: the title and the limitations associated with the methodology, respectively.

First, the title does not say that suicidal behaviour was changed. The title states that the aim of the intervention was 'to reduce subsequent suicidal ideation and behaviour'. Moreover, we insisted on the term 'exploratory' remaining in the title against the suggestion of a reviewer and explicitly did not present the 6-month outcomes as main findings.

Second, House \& Owens are correct that there are methodological limitations to the study, but these are recognised in the paper. Indeed, the final sentence of our conclusion chimes with the points they raise: 'Further research is required to replicate the findings with a more complete data-set and objective outcome measures'. In total, we highlighted six points of caution with respect to the interpretation of the findings. Methodological limitations are inherent in exploratory studies of this kind, but we endeavoured to address them by making weaknesses explicit and adjusting analyses to mitigate the effects of limitations as far as possible.

We believe our approach of last observation carried forward is a good example of adjustment to the limitation of missing data here. As House \& Owens note, the observation carried forward method is not perfect and may introduce bias, which we assumed most readers would be well aware of and therefore did not flag in our 'limitations' section. In terms of the present study, the observation carried forward was pre-intervention, rather than post-intervention. Given that our randomisation check demonstrated no differences between conditions pre-intervention, any score carried forward would, if anything, bias results against finding an effect of the intervention. Had we chosen instead to measure outcomes immediately post-intervention and carried these observations forward, then the findings would have been biased in favour of finding an effect of the intervention. We would therefore argue that, cognisant of the limitations of last observation carried forward per se, we adopted the most rigorous approach possible within the given context (a busy emergency department in a low- and middle-income country).

Another example of our attempt to make the limitations of our study explicit concerns the points we make about the measurement of the main outcome variable. It was not possible to record re-attendance following non-fatal self-harm, and so we had to rely on the use of the Suicidal Behaviors Questionnaire a self-report measure of suicidal ideation and behaviour. In the discussion, we state that it would be useful to have a more objective outcome measure, such as future hospital admissions, although this is not currently possible in the Malaysian context'. It behoves us to conduct research such as ours in low- and middle-income countries, even if it can only be exploratory at the present time.

In sum, we stand by our conclusion that this paper details a promising approach to intervention that is worthy of further research.

Christopher J. Armitage, Professor of Health Psychology, University of Manchester. Email: chris.armitage@manchester.ac.uk; Wirda Abdul Rahim, Richard Rowe, Department of Psychology, University of Sheffield; Rory C. O'Connor, Institute of Health and Wellbeing, University of Glasgow

doi: 10.1192/bjp.209.4.351

\section{Evolutionary theories in disordered eating psychopathology}

It was disappointing to find that the editorial by Murray et al ${ }^{1}$ 'Evolving eating disorder psychopathology: conceptualising muscularity-oriented disordered eating' made no mention of evolutionary formulations of eating disorders despite the somewhat suggestive title. This is a significant omission, as evolutionary theories provide a cogent explanatory framework not only for the newly described male variant of eating disorder referred to in the article but also for eating disorders as a whole. Also, the editorial's title promised to deliver a new conceptual framework for eating disorders in the light of this new variant, but all it did was produce the familiar tautology regarding cultural body ideals that has had such poor explanatory value in the case of female eating disorders. The formulation's lack of predictive power has meant that little, if any, progress has been made in the past few decades in researching the aetiology of these conditions despite the huge quantities of data collected. The weakness of the cultural norm (of desirable shape, beauty etc.) formulation is evident from the fact that it attempts to explain the phenomena of eating disorder by assigning causation to the very thing it is meant to explain (e.g. the drive for thinness in women or muscularity in men).

Hence, if these societal ideals are arbitrary and not related to any underlying biologically based motives, we should observe the reverse pattern in some societies (e.g. more men than women wishing to be thinner and more women than men wishing to be more muscular), but there is no evidence of such a scenario.

The sexual competition hypothesis $(\mathrm{SCH})$ for eating disorders $^{2}$ is an evolutionary formulation that can help answer the 'why' question that has so far defeated mainstream, non-evolutionary theories. It proposes that all eating disorders stem from the phenomenon of intrasexual competition taken to pathological extremes. In the case of females, competition is 
primarily through the display of signs of youth (where thinness is a marker for youth). Youth is a major determinant of reproductive potential in females, whereas men compete among themselves through the display of physical dominance and prowess, which is enhanced through the size and muscularity of the upper body. The increase in the rates of eating disorders in the past several decades in Western and Westernised countries is explained by the 'mismatch' between the human psychological systems for mate attraction and retention and for competition with same-sex rivals that evolved in small-scale societies on the one hand, and the novel realities of the modern urban Western environment on the other.

The SCH has been supported by findings of studies of disordered eating in non-clinical populations (female undergraduates) both in the USA ${ }^{3}$ and the UK. ${ }^{4}$ Further support for predictions made by the $\mathrm{SCH}$ came from another US study of disordered eating in a non-clinical population of male and female homosexual and heterosexual participants. ${ }^{5}$

The consistency of the clinical features of eating disorders across different high-income countries, as well as their persistence (or even rising levels), argues strongly against the arbitrary cultural standard view dominant in eating disorder circles and in favour of a model in which these disorders are the outcome of a gene-environment interaction.

1 Murray SB, Griffiths S, Mond JM. Evolving eating disorder psychopathology: conceptualising muscularity-oriented disordered eating. Br J Psychiatry 2016, 208: $414-5$.

2 Abed, R. The sexual competition hypothesis for eating disorders. $\mathrm{Br} J \mathrm{Med}$ Psychol 1998; 71: 525-47.

3 Faer L, Hendriks A, Abed R, Figueredo AJ. The evolutionary psychology of eating disorders: female competition for mates or status? Psychol Psychother 2005; 78: 397-417.

4 Abed R, Mehta S, Figueredo AJ, Aldridge S, Balson H, Meyer C, et al. Eating disorders and intrasexual competition: testing an evolutionary hypothesis among young women. Scientific World J 2012; 2012: 290813.

5 Li NP, Smith AR, Griskevicius V, Cason MJ, Bryan A. Intrasexual competition and eating restriction in heterosexual and homosexual individuals. Evol Hum Behav 2010; 31: 365-72.

Riadh T. Abed, Psychiatrist, Mental Health Tribunals, Ministry of Justice, England. Email: abedrt@btinternet.com

doi: $10.1192 / \mathrm{bjp} .209 .4 .351 \mathrm{a}$

Authors' reply: We thank Dr Abed for his comment on our recent editorial but dispute the implication that delineating the symptom profiles of male versus female eating disorder pathology neglects the aetiological precursors of eating disorders. On the contrary, careful delineation of the spectrum of disordered eating that occurs at the population level is essential in developing comprehensive aetiological frameworks. For many years, the male experience of disordered eating has been under-represented in empirical and clinical research. The purpose of our editorial ${ }^{1}$ was to highlight both the male experience of eating disorder pathology, and the historical failure to recognise and index this pathology.
Although our piece was not intended to address considerations of aetiology, it is important to note that there is strikingly little evidence to support the sexual competition hypothesis as an aetiological framework for muscularity-oriented disordered eating, as proposed by Abed. Indeed, much of the work cited by Abed was conducted in female samples, with a consequent focus on the drive for thinness, and the study mentioned by Abed, ${ }^{2}$ in which the sexual competition hypothesis was assessed in nonclinical male and female populations, is merely another example of male eating disorder pathology being assessed through the lens of female-oriented measurement tools. As such, the statement that the sexual competition hypothesis provides a cogent framework in accounting for the aetiology of muscularity-oriented disordered eating goes beyond what the current evidence base permits. The propensity to extrapolate findings from thinness-oriented studies of eating disorder pathology to males is the very problem which our editorial aimed to highlight.

In our view, the assertion that evolution-instilled competition for mates provides a framework for understanding the aetiology of eating disorder pathology is problematic for at least two reasons. First, eating disorders are associated with a substantially elevated risk of mortality, ${ }^{3}$ a distinct evolutionary disadvantage. Second, clinical eating disorder pathology is frequently characterised by a reduction in libido and active avoidance of sexual relationships, ${ }^{4}$ observations that are at odds with the purported goal of dietary restraint. Additionally, this framework offers little insight into how to distinguish those who develop clinical eating disorders from the far greater numbers of individuals who engage in purposeful dietary restraint with the goal of modifying their shape or weight, many of whom presumably desire a mate. No doubt a more comprehensive aetiological framework for eating disorder pathology needs to take better account of the reciprocal influences of a broad range of factors, but to say that 'these disorders are the outcome of a gene-environment interaction' is a diffuse and perhaps oversimplified statement which is likely more harmful than helpful.

1 Murray SB, Griffiths S, Mond JM. Evolving eating disorder psychopathology: conceptualising muscularity-oriented disordered eating. Br J Psychiatry 2016; 208: $414-5$.

2 Li NP, Smith AR, Griskevicius V, Cason MJ, Bryan A. Intrasexual competition and eating restriction in heterosexual and homosexual individuals. Evol Hum Behav 2010; 31: 365-72.

3 Arcelus J, Mitchell AJ, Wales J, Nielsen S. Mortality rates in patients with anorexia nervosa and other eating disorders: a meta-analysis of 36 studies. Arch Gen Psychiatry 2011; 68: 724-31.

4 Pinheiro AP, Raney TJ, Thornton LM, Ficther MM, Berrettini WH, Goldman D, et al. Sexual functioning in women with eating disorders. Int J Eat Disord 2010; 43: 123-9.

Stuart B. Murray, Department of Psychiatry, University of California, San Francisco, 401 Parnassus Avenue, San Francisco, California, USA. Email: drstuartmurray@ gmail.com; Scott Griffiths, School of Psychology, Australian National University, Canberra, Australia; Jonathan M. Mond, Department of Psychology, Macquarie University, Sydney, Australia

doi: 10.1192/bjp.209.4.352 